\title{
A Simple Electrostatic Device for Eliminating Tobacco Sidestream Smoke to Prevent Passive Smoking
}

\author{
Yoshinori Matsuda ${ }^{1, *}$, Koji Kakutani ${ }^{2,3}$, Teruo Nonomura ${ }^{1}$, Yoshihiro Takikawa ${ }^{4}$, \\ Kiyotsugu Okada ${ }^{5}$, Manabu Shibao ${ }^{5}$, Kazuhiro Miyama ${ }^{6}$, Shinya Yokoo ${ }^{6}$, Shin-ichi Kusakari ${ }^{7}$ \\ and Hideyoshi Toyoda ${ }^{7}$ \\ 1 Laboratory of Phytoprotection Science and Technology, Faculty of Agriculture, Kindai University, \\ Nara 631-8505, Japan; nonomura@nara.kindai.ac.jp \\ 2 Pharmaceutical Research and Technology Institute, Kindai University, Osaka 577-8502, Japan; \\ kakutani@kindai.ac.jp \\ 3 Anti-Aging Centers, Kindai University, Osaka 577-8502, Japan \\ 4 Plant Center, Institute of Advanced Technology, Kindai University, Wakayama 642-0017, Japan; \\ takikawa@waka.kindai.ac.jp \\ 5 Research Institute of Environment, Agriculture and Fisheries, Osaka 583-0862, Japan; \\ okada@mbox.kannousuiken-osaka.or.jp (K.O.); shibao@mbox.kannousuiken-osaka.or.jp (M.S.) \\ 6 T-CUBE, Earth Environmental Service Co., Ltd., Osaka 567-0085, Japan; \\ miyama-kazuhiro@earth-kankyo.co.jp (K.M.); yokoo-shinya@earth-kankyo.co.jp (S.Y.) \\ 7 Research Association of Electric Field Screen Supporters, Nara 631-8505, Japan; \\ kusakari@mbox.kannousuiken-osaka.or.jp (S.K.); toyoda@nara.kindai.ac.jp (H.T.) \\ * Correspondence: ymatsuda@nara.kindai.ac.jp; Tel.: +81-742-43-5223
}

Received: 16 June 2018; Accepted: 20 July 2018; Published: 22 July 2018

\begin{abstract}
An electrostatic apparatus was constructed to capture tobacco sidestream smoke. This apparatus consisted of a perforated polypropylene plate with metal spikes and a grounded metal net arrayed in parallel at a defined interval. Spikes were negatively charged to positively polarize the net and an electric field was formed between the opposite charges of the spike tips and the grounded net. Discharge from the spike tips occurred, which depended on the pole distance and the voltage applied to the spikes. At lower voltages $(<12.1 \mathrm{kV})$ that do not cause arc discharge from the tips, a corona discharge occurred with the generation of an ionic wind from the spiked plate to the net. This discharge increased in direct proportion to the applied voltage and relative humidity, while a larger corona discharge generated a stronger ionic wind. The ionic wind involved negative ions and the number of negative ions in the wind increased with increasing applied voltage. The optimal voltage $(10 \mathrm{kV})$ generated sufficient negative ions to ionize smoke particles in the electric field, before the ionized smoke particles were successfully captured by the oppositely charged metal net. Thus, this study provides an experimental basis for the practical application of an electrostatic-based method to prevent the production of tobacco sidestream smoke that leads to passive smoking by non-smokers.
\end{abstract}

Keywords: electrostatic smoke eliminator; corona discharge; ionic wind; negative ions; fine smoke particles

\section{Introduction}

Passive smoking is the inhalation of environmental tobacco smoke (i.e., of second-hand smoke by persons that do not intend to smoke). Tobacco sidestream smoke permeates the environment, which is inhaled by people within that environment. Exposure to second-hand tobacco smoke can cause disease, disability and death [1]. The health risks of second-hand smoke are a matter 
of scientific consensus. In fact, second-hand smoke causes many of the same diseases as direct smoking, including cardiovascular diseases, lung cancer and respiratory diseases [2]. In particular, pregnant females who are exposed to environmental tobacco smoke have a higher risk of delivering children with congenital abnormalities, longer lengths, smaller head circumferences and low birth weights [3]. In addition, there is a sufficient evidence to infer a causal relationship between exposure to second-hand smoke and sudden infant death syndrome [4]. Thus, second-hand smoke is the most significant indoor pollutant for humans. These risks have served as a major motivation for smoke-free laws in workplaces and indoor public places. In this present study, we aimed to physically eliminate tobacco smoke in order to keep indoor air as clean as possible. We achieved this by developing an electrostatic air purification device that prevents tobacco sidestream smoke from entering public spaces and thus, affecting non-smokers.

Applied electrostatic engineering has served as the academic and technical basis for various methods that have been implemented to successfully manage pathogens and insect pests affecting agricultural crops during various stages of crop production and preservation. There are diverse ways in which electrostatic principles have been applied, which include the following: capturing spores and insects by exploiting the attractive forces generated in a static electric field (without electric discharge) [5-10]; repelling insects according to their aversion to the electric field [5,6,11,12]; disinfecting bacterial and fungal pathogens using ozone produced through streamer discharge [13]; and instantaneously dislodging fungal pathogens from plants through exposure to a plasma stream produced via a corona discharge in the electric field [14]. Based on these successful applications, we propose an electrostatic apparatus to ionize fine smoke particles with negative ions produced within an electric field.

The main purpose of our research was to present a practical apparatus with a simple structure, which can be constructed easily at a low cost and functions according to electrostatic principles. Our apparatus consists of a pair of perforated polypropylene plates (PPs) with negatively charged spikes and a positively polarized grounded metal net. The opposite surface charges of the spikes and metal net form an electric field that generates an ionic wind, which negatively ionizes smoke particles. We optimized its configuration by determining the most appropriate voltage at a definite pole distance for optimal capture of the fine tobacco sidestream particles blown inside the electric field.

\section{Materials and Methods}

\subsection{Construction of Electrostatic Smoke Eliminator}

A perforated PP (insulator; $40 \times 35 \mathrm{~cm}, 2$-mm thick) with octagonal holes at constant intervals and a stainless net (SN) (conductor; $40 \times 35 \mathrm{~cm}, 1.5 \mathrm{~mm}$-mesh) were used to construct an electrostatic smoke eliminator (ESE; Figure 1A). Fifty copper pins (conductor; pinhead with a diameter of $10 \mathrm{~mm}$ and needle with a length of $8 \mathrm{~mm}$ ) were stuck to the PP with conductive double-side adhesive tape (CT) to construct a spiked PP (see Figure 2A). The CT was connected to a direct-current voltage generator (current limit of $10 \mathrm{~mA}$; AMA-20K10NKBX1, Max-Electronics, Tokyo, Japan) and negatively charged with different voltages. The SN was arranged in parallel with the spiked PP at a defined interval $(10 \mathrm{~mm})$ and linked to a ground line. The SN and spiked PP were secured in a polyvinyl chloride frame. The negative surface charges on the spikes caused electrostatic induction in the $\mathrm{SN}$, creating an opposite surface charge on the PP-side surface of the SN. As a result, an electric field formed between these opposite charges (Figure 1B). The transfer of free electrons from the PP-side to the SN-side ground was measured using two galvanometers (PC7000; Sanwa Electric Instrument, Tokyo, Japan) integrated into the grounded lines. 
A

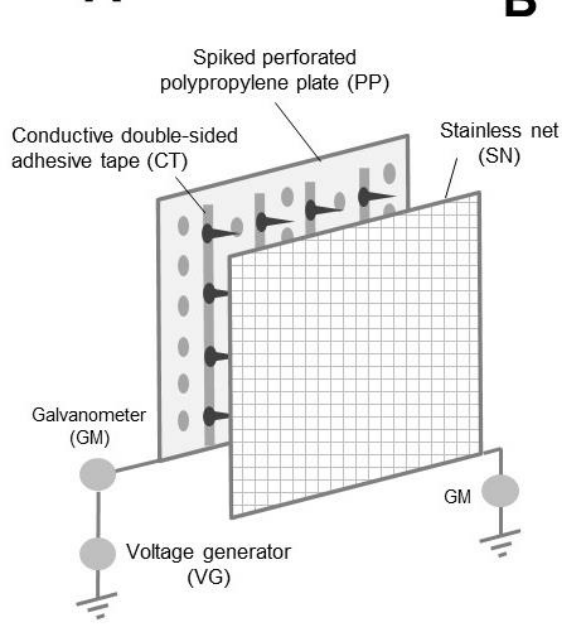

C

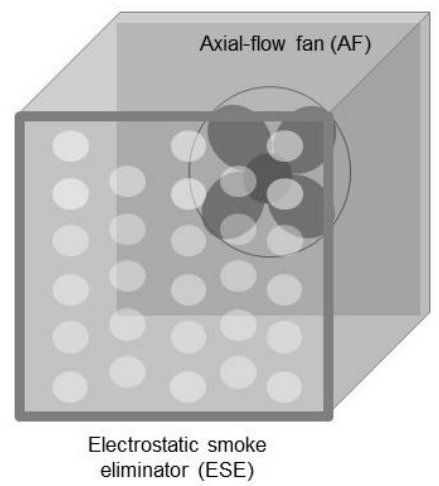

B

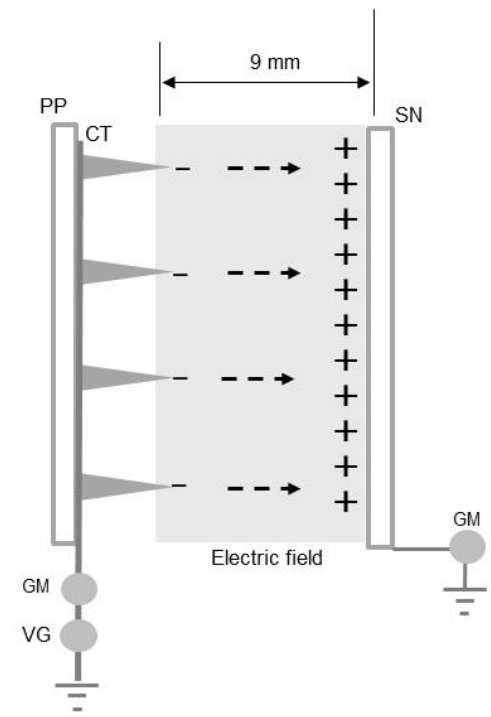

D

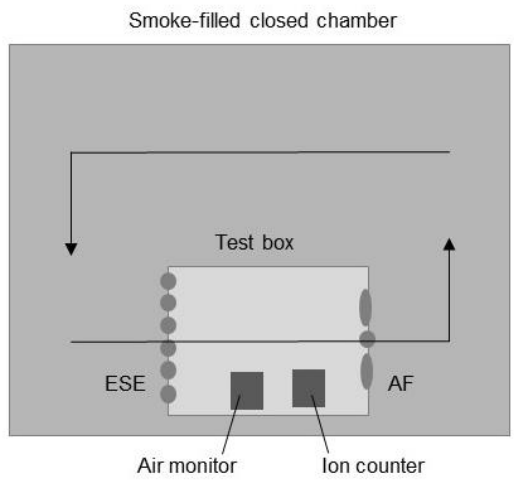

Figure 1. Structure (A) and cross-sectional view (B) of the spiked perforated polypropylene plate and stainless net of the electrostatic smoke eliminator (ESE). A transparent acrylic cubic box (test box) furnished with the ESE (C). The test box placed in a temperature- and humidity-controlled chamber for a particle-capturing assay (D). Dashed (B) and solid (D) arrows show the direction of ionic wind and the airflow, respectively.
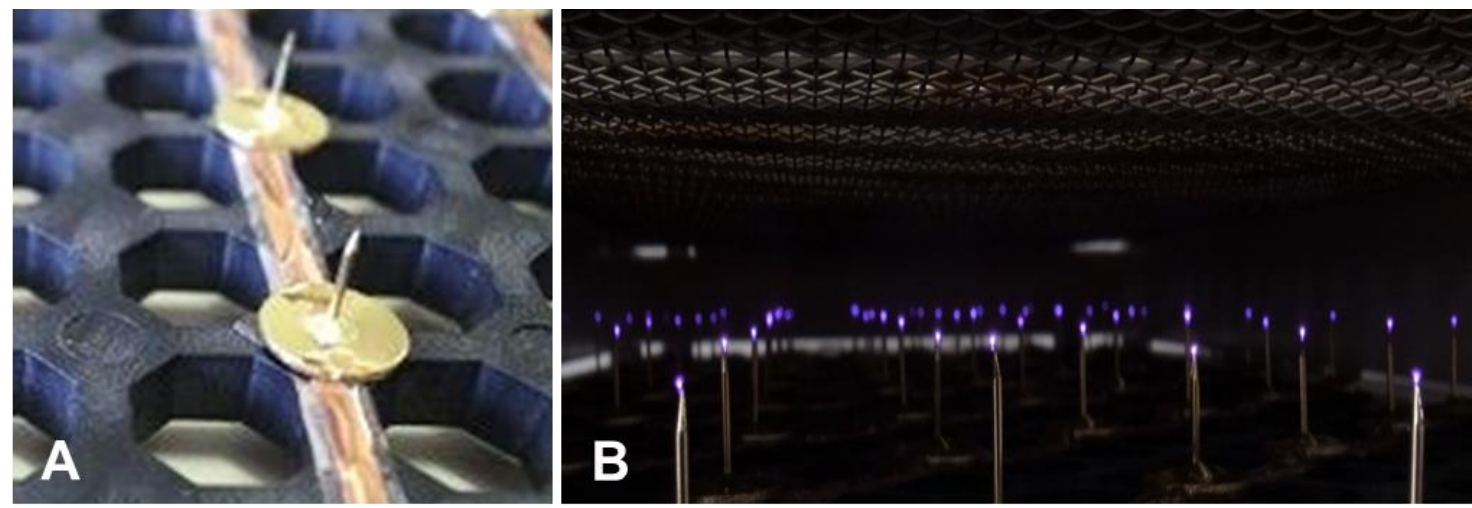

Figure 2. Pin-spikes adhered to the perforated PP (A) and corona discharge glow from the tips of the pin-spikes photographed in a dark field (B). 


\subsection{Smoke Capturing Assay}

Prior to the smoke capturing assay, the pin-spikes of the ESE were negatively charged with 1-15 kV to determine the voltage range that would cause a mechanical discharge in the electric field. Namely, this mechanical discharge is an arc discharge from the pin tips of the PP to the SN. In the smoke capturing assay, the spikes of the PP were negatively charged with different voltages $(1-12 \mathrm{kV})$ that caused no mechanical discharge. This allows us to examine the ability of the ESE to capture fine particles in smoke. In this assay, cigarettes (Japan Tobacco, Tokyo, Japan) were used to generate smoke, with the cigarettes having emitted smoke particles with a diameter of $0.01-0.5 \mu \mathrm{m}$ when burned. A transparent acrylic cubic box (side length of $35 \mathrm{~cm}$ ) was furnished with the ESE on one side and an axial-flow fan (blade length of $8 \mathrm{~cm}$ ) on the other side (Figure 1C). This test box was placed inside a closed chamber controlled at $25^{\circ} \mathrm{C}$ and $60 \%$ relative humidity (RH; Figure 1D). Burning cigarettes were placed inside the chamber and the internal air was circulated at a particular wind speed (equivalent to the speed of the ionic wind generated by each applied voltage) by operating the axial-flow fan of the test box to achieve constant production of smoke. The airflow speed was measured at the surface of the ESE using a sensitive anemometer (Climomaster 6533; Kanomax, Tokyo, Japan). The particle density in the circulated air was measured using an air quality monitor (DC1100 PRO; Sato Shouji Inc., Kawasaki, Japan; detectable particle range: 0.5-2.5 $\mu \mathrm{m}$ in diameter) placed inside the test box. In this monitor, scattered light was generated when the particles passed through the light in the detector cell, which were detected by the photo detector and converted to electrical signals (light-scattering method). The size of the electrical signals represented the particle size and the frequency of scattered-light detection represented the particle count. When the particle density reached a certain level $\left(4.0 \times 108\right.$ particles $\left./ \mathrm{m}^{3}\right)$, we removed the smoke source, before charging the ESE for $120 \mathrm{~s}$ with negative voltages in order to determine the voltage range required for capturing all of the particles passing through the ESE. The non-charged box was used as a negative control. The number of particles in the charged box was expressed as a percentage relative to that of the non-charged control. Smoke attraction was also recorded using a digital electro-optical system (EOS) camera (Canon, Tokyo, Japan).

\subsection{Detection of Corona Discharge, Ionic Wind and Negative Ions in the Electric Field}

A corona discharge (continuous corona current) [15], which was constantly generated from the spike tips, was measured in the voltage range of $6-10 \mathrm{kV}$ and exerted an attractive force on the smoke particles. The electric current from the corona discharge was recorded with a built-in galvanometer, while the glow of the corona discharge was photographed during a long exposure in a dark field. The speed of the airflow (ionic wind) from the PP spike tips to the SN generated by the corona discharge was measured at the outside surface of the SN using a high-sensitivity anemometer (Climomaster 6533; Kanomax, Tokyo, Japan) and the volumetric flow rate $\left(\mathrm{m}^{3} / \mathrm{min}\right)$ was calculated using the equation $\mathrm{Q}\left(\mathrm{m}^{3} / \mathrm{min}\right)=\mathrm{V}\left(\mathrm{m}^{2}\right) \times \mathrm{A}(\mathrm{m} / \mathrm{s}) \times 60(\mathrm{~s})$. The number of negative ions involved in the airflow was estimated with a Gerdien atmospheric ion counter (NKMH-103; Hokuto Electronic, Hyogo, Japan) [16] placed in the test box. Experiments were conducted under the conditions described earlier.

\subsection{Assay of Effect of Humidity Change on the Trapping of Smoke Particles}

Different temperature and RH conditions were applied to achieve different values $\left(0.002-0.032 \mathrm{~kg} / \mathrm{m}^{3}\right)$ of volumetric humidity (VH; absolute humidity). VH was calculated according to three parameters (temperature, RH and barometric pressure) using an online RH to VH converter (https: / / planetcalc. com/2167/). The ESE was negatively charged with different voltages under different humidity conditions, before the electric current from the corona discharge was recorded using the method mentioned earlier. 


\subsection{Statistical Analysis}

All experiments were repeated five times and the data are presented as means \pm standard deviation (SD). Significant differences were analyzed using Tukey's method, with a $p$-value $<0.05$ considered to be statistically significant. We analyzed the correlation between the applied voltage and the corona discharge, the volumetric flow rate of the ionic wind, or number of negative ions; and between the negative ion generation and smoke particle capture. The regression line of these correlations was provided from the plots of values obtained by measurements.

\section{Results and Discussion}

In our previous research, we developed an electric field between insulated conductor wires (ICWs) and a grounded non-insulated metal net, or between ICW(-) and ICW (+), to trap insect pests. In these systems, ICW $(-)$ pushed free electrons from the surface cuticle layers (conductor) of the insects released near ICW $(-)$ to give insects a net positive charge $[8,9,17]$, while insects near ICW $(+)$ gained a net negative charge with the addition of free electrons to the cuticle layer [7]. Ultimately, the negatively and positively electrified insects were attracted to oppositely charged electrodes. In a subsequent study [18], we presented an additional explanation of the fungal spore attraction, stating that this was due to the dielectrophoretic movement of spores subjected to a non-uniform electric field between ICW(-) and ICW(+). Dielectrophoresis is a phenomenon in which a force is exerted on a dielectric particle (oppositely polarized particle) in a non-uniform electric field [19]. This force does not require the particle to be charged because all particles exhibit dielectrophoretic activity in the presence of a non-uniform electric field. According to the dielectrophoresis theory, the polarization of the particle (spore) relative to the surrounding electric field changes along the gradient of the electric field strength. This variable polarization enables the particle to move toward the electrodes. In the present study, we paid special attention to the electric field that was produced between the two non-insulated conductor poles, in which the electrostatic attractive force between the negatively ionized particles in smoke and the oppositely electrified pole can effectively capture tobacco smoke particles.

\subsection{Capture of Fine Particles of Cigarette Smoke by ESE}

Figure 3 shows the percentage of fine tobacco smoke particles trapped by the ESE that were charged at different voltages (1-12 kV) and causing no mechanical discharge. The number of detector-recorded fine particles decreased with applied voltage. In the electric field, a particle was subjected to an electrostatic attractive force and a force produced by the airflow, with the direction of the particle's motion being determined by the combined vector of these two forces. The ESE trapped all smoke particles in the electric field when the voltage applied to the electrodes exceeded $10 \mathrm{kV}$. However, at lower voltages, the particles passed through the SN. Under these conditions, the attractive force produced by the ESE was weaker than the airflow force. These findings indicate that a reduction in trapping force increased as the voltage decreased. To demonstrate this visually, Video S1 in Supplementary Material shows that the ground net of the ESE can capture the fine particles in smoke blown toward the ESE. In the video, the electrostatic barrier completely prevents the smoke from passing through the ESE at a sufficient charge $(10 \mathrm{kV})$.

\subsection{Involvement of Negative Ions in Smoke Attraction in the Electric Field}

In the electrostatic configuration of the present apparatus, high voltages produced through a Cockcroft circuit [20] in the voltage generator were used to channel electricity from the ground to the spike tips of the PP, while their negative surface charge pushed the free electrons of the $\mathrm{SN}$ to the ground to create an opposite pole (see Figure 1B). In this study, we formed an electric circuit in which the electricity (free electrons) moved from ground to ground when the discharge of the negative poles (spike tips) occurred. The pole distance was fixed, while the voltage was changed to determine the voltage ranges causing mechanical and corona discharge in the electric field. 


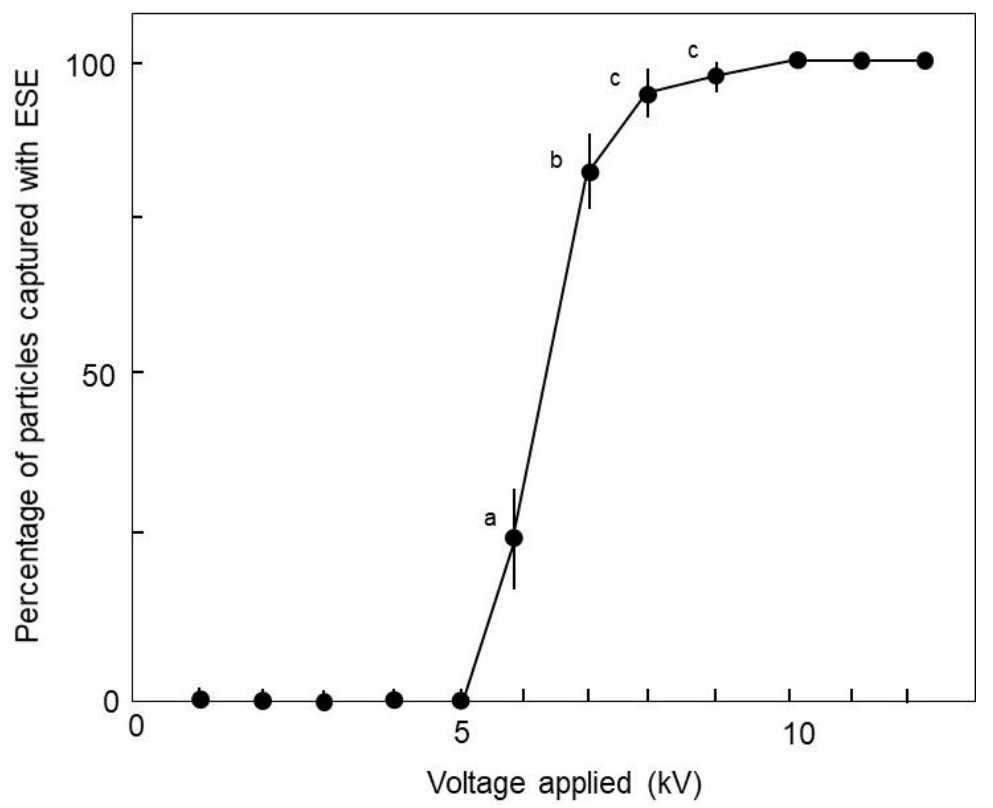

Figure 3. Purification of fine particles of tobacco smoke by the ESE charged with different voltages. The experiment was conducted at $25{ }^{\circ} \mathrm{C}$ and $60 \%$ relative humidity (RH). The mean and standard deviation were calculated from five replicates. The different letters $(\mathrm{a}-\mathrm{c})$ on the plots indicate significant differences $(p<0.05)$ according to Tukey's method.

In the preliminary experiment, we determined the voltage that would cause mechanical discharge in the electric field. The spike tips on the PP generated mechanical discharge (arc discharge). As the voltage applied to the spikes was increased, the apparatus eventually caused a mechanical discharge at $>12 \mathrm{kV}$, which occurred regardless of the changes in temperature and $\mathrm{RH}$. This result implied that at $\leq 12 \mathrm{kV}$, the ESE could be operated without causing a mechanical discharge, even with any changes in environmental conditions.

A corona discharge will occur when the strength (potential gradient) of the electric field around a pointed metal conductor is high enough to form a conductive region but not high enough to cause electrical breakdown or arcing to nearby objects [15]. The present electrostatic device was configured so that the two non-insulated conductor nets and spikes faced each other to produce an electric field. In this electric field, a corona discharge constantly occurred from the spike tips of the PP to the SN within the voltage range causing no arc discharge (Figure 2A,B). Figure $4 \mathrm{~A}$ shows the relationship between the applied voltage and the corona discharge generation. In the present apparatus, the electric current produced by this discharge became greater in direct proportion to increases in the applied voltage in the range of $6-10 \mathrm{kV}$, which allowed us to capture $0.1-100 \%$ of smoke particles (see Figure 3).

An ionic wind is defined as the airflow induced by electrostatic forces linked to corona discharge, which arises at the tips of some sharp conductors (such as points or blades) that are subjected to high voltage relative to the ground [15]. The present study showed a clear correlation between the applied voltage and generation of ionic wind as the generation increased in direct proportion to the applied voltage (Figure 4B). Measurements with an ion detector confirmed that larger voltages produced a stronger ionic wind involving more ions (Figure 4C). The ionic wind was directed from the pointed tip of the PP toward the SN (see Figure 1B) as reported in previous work [21]. The ionic wind produced by the present apparatus was sufficiently strong $(1.5-2.0 \mathrm{~m} / \mathrm{s})$ to bring outside air into the electric field of the ESE. Figure 5 shows the positive correlation between negative ion generation and the trapping of fine smoke particles in the electric field of the ESE. These results strongly support the involvement of negative ions in the electrostatic attraction of smoke particles in the electric field of the ESE. Thus, 
the results met our expectation that negative ions can ionize smoke particles to generate an attractive force toward the opposite charge on the metal net.

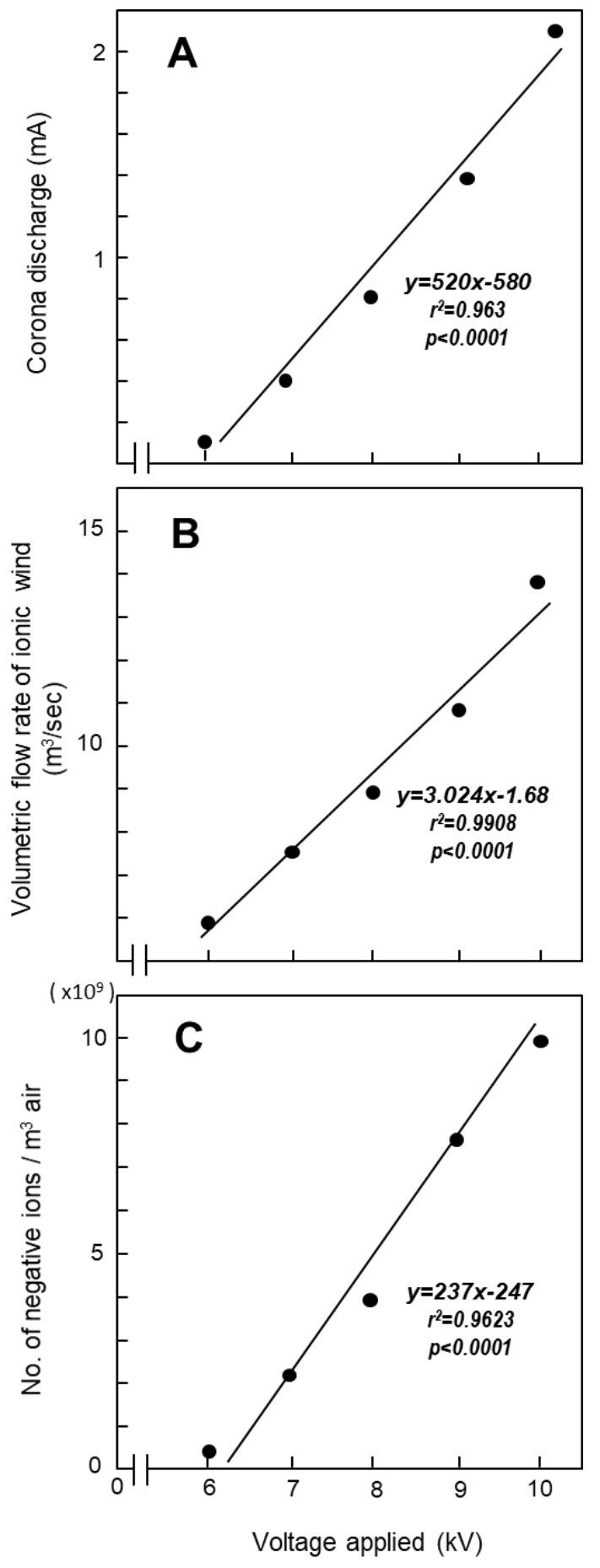

Figure 4. Relationship between the applied voltage and corona discharge (A), volumetric flow rate of ionic wind (B) and negative ion generation (C) in the electric field of the ESE. The experiment was conducted at $25^{\circ} \mathrm{C}$ and $60 \% \mathrm{RH}$. 


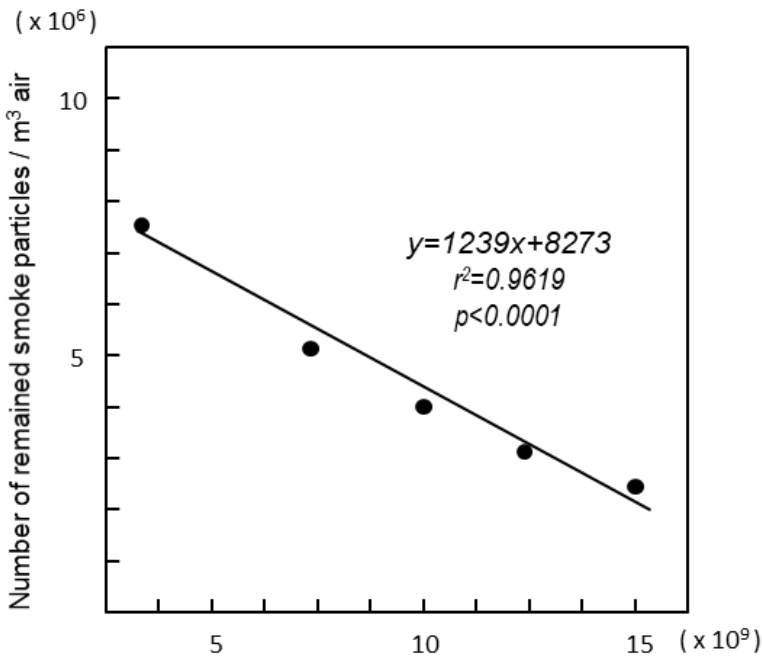

Figure 5. Correlation between negative ion generation and trapping of fine smoke particles in the electric field of the ESE.

\subsection{Effect of Air Conductivity on the Current Generation by Corona Discharge}

In addition to the voltage applied to the electrode and the electrode distance (pole distance), the air conductivity between the electrodes can affect the flow (electric current) of the accumulated electricity [15]. Air conductivity changes in response to changes in water-vapor concentration in the air as the air conductivity increases (i.e., higher electrical transfer) as VH (absolute humidity) increases [22]. In the present study, we examined the effect of humidity changes on current generation by corona discharge in the electric field of the ESE that was negatively charged with different voltages (Table 1). At all voltages, the electric current increased with increasing humidity. At $\geq 8 \mathrm{kV}$, the increase in current was insignificant. In fact, the ESE exhibited $100 \%$ capture of smoke particles at $10 \mathrm{kV}$, which occurred regardless of the change in humidity. These results imply that the smoke-capturing function of the ESE works in all humidity conditions under the several specific voltages tested.

In summary, our novel ESE is a promising device for creating a smoke-free space for non-smokers and preventing passive smoking. The device can be constructed at a low cost due to its very simple structure and operates in a stable manner regardless of changes in environmental conditions, such as temperature and humidity.

Table 1. Effect of humidity changes on current generation by corona discharge in the electric field of the ESE that was negatively charged with different voltages.

\begin{tabular}{|c|c|c|c|c|c|c|c|c|c|c|c|c|}
\hline \multirow{2}{*}{$\begin{array}{c}\text { Volumetric } \\
\text { Humidity }\left(\mathrm{g} / \mathrm{m}^{3}\right)\end{array}$} & \multirow{2}{*}{$\begin{array}{c}\text { Temperature } \\
\left({ }^{\circ} \mathrm{C}\right)\end{array}$} & \multirow{2}{*}{$\begin{array}{c}\text { Relative } \\
\text { Humidity (\%) }\end{array}$} & \multicolumn{10}{|c|}{ Electric Current $(\mu \mathrm{A})$} \\
\hline & & & 4 & & 6 & & 8 & & 10 & & $12(\mathrm{kV})$ & \\
\hline 0.004 & 15 & 30 & 0.1 & a & $60.6 \pm 3.8$ & $\mathrm{a}$ & $796.8 \pm 3.3$ & a & $2195.2 \pm 5.8$ & $\mathrm{a}$ & $3996.4 \pm 5.7$ & $\mathrm{a}$ \\
\hline 0.008 & 15 & 60 & $8.2 \pm 1.9$ & b & $101.8 \pm 12.4$ & b & $802.6 \pm 4.3$ & a & $2202.6 \pm 7.2$ & a & $4004.2 \pm 5.8$ & a \\
\hline 0.018 & 25 & 80 & $40.0 \pm 9.3$ & c & $185.4 \pm 19.6$ & c & $804.6 \pm 6.5$ & $\mathrm{a}$ & $2207.8 \pm 10.7$ & a & $4006.6 \pm 9.1$ & a \\
\hline 0.024 & 30 & 80 & $42.2 \pm 13.0$ & c & $188.2 \pm 28.1$ & c & $805.6 \pm 6.5$ & a & $2211.8 \pm 12.6$ & a & $4008.4 \pm 8.4$ & a \\
\hline 0.032 & 35 & 80 & $42.8 \pm 14.7$ & c & $191.6 \pm 24.9$ & c & $806.4 \pm 8.6$ & a & $2217.2 \pm 12.7$ & a & $4012.6 \pm 13.6$ & a \\
\hline
\end{tabular}

Means and standard deviations were calculated from five replicates. Letters (a-c) indicate significant differences within each column $(p<0.05)$ according to Tukey's method.

\section{Conclusions}

The primary contribution of this work was the use of basic electrostatics for improving the human environment. The ESE is a unique product developed for this purpose. The structure of the ESE is simple and no special technique is required for its construction. The ESE can be operated easily to capture fine particles in tobacco smoke by the use of an attractive force between the negatively 
ionized particles and the oppositely charged mechanical pole. Another advantage of the ESE is its structural safety. An electric field was produced inside the ESE, while the outer surfaces of the spiked plate and grounded net possessed no charge so the net surface can be safely touched. An additional safeguard was the current limiter (maximum limit of $10 \mathrm{~mA}$ ) integrated into the electric circuit of the voltage generator, which automatically switched off the generator if excess current was generated by an unexpected event. This work demonstrates that the proposed ESE can easily eliminate fine PM from living spaces in order to improve the comfort of occupants and minimize the likelihood of air quality-related health problems.

Supplementary Materials: The following are available online at http:/ / www.mdpi.com/2410-390X/2/3/13/s1, Video S1: Trapping of tobacco smoke by the ESE. In this video, the voltage generators were switched on and off repeatedly, as indicated by the switching sound. Note that the smoke was completely prevented from passing through the ESE when a voltage of $10 \mathrm{kV}$ was applied.

Author Contributions: Conceptualization, Y.M., K.K., and H.T.; Methodology, K.K., T.N., Y.T.; Validation, K.K., T.N., Y.T., K.O., M.S., K.M., and S.Y.; Investigation, K.K. and S.K.; Data Curation, K.K., T.N., Y.T., K.O., M.S., K.M. and S.Y.; Writing-Original Draft Preparation, Y.M.; Writing-Review \& Editing, H.T.; Visualization, Y.M.; Supervision, Y.M., S.K., and H.T.; Project Administration, S.K. and H.T.

Funding: This work was supported by JSPS KAKENHI Grant Number 16K00803.

Conflicts of Interest: The authors declare no conflict of interest.

\section{References}

1. Cao, S.; Yang, C.; Gan, Y.; Lu, Z. The health effects of passive smoking: An overview of systematic reviews based on observational epidemiological evidence. PLoS ONE 2015, 10, e0139907. [CrossRef] [PubMed]

2. Dinas, P.C.; Koutedakis, Y.; Flouris, A.D. Effects of active and passive tobacco cigarette smoking on heart rate variability. Int. J. Cardiol. 2013, 163, 109-115. [CrossRef] [PubMed]

3. Cui, H.; Gong, T.T.; Liu, C.X.; Wu, Q.J. Associations between passive maternal smoking during pregnancy and preterm birth: Evidence from a meta-analysis of observational studies. PLoS ONE 2016, 11, e0147848. [CrossRef] [PubMed]

4. Saulyte, J.; Regueira, C.; Montes-Martínez, A.; Khudyakov, P.; Takkouch, B. Active or passive exposure to tobacco smoking and allergic rhinitis, allergic dermatitis, and food allergy in adults and children: A systematic review and meta-analysis. PLoS Med. 2014, 13, e1001939. [CrossRef] [PubMed]

5. Matsuda, Y.; Ikeda, H.; Moriura, N.; Tanaka, N.; Shimizu, K.; Oichi, W.; Nonomura, T.; Kakutani, K.; Kusakari, S.; Higashi, K.; et al. A new spore precipitator with polarized dielectric insulators for physical control of tomato powdery mildew. Phytopathology 2006, 96, 967-974. [CrossRef] [PubMed]

6. Matsuda, Y.; Nonomura, T.; Kakutani, K.; Takikawa, Y.; Kimbara, J.; Kasaishi, Y.; Kusakari, S.; Toyoda, H. A newly devised electric field screen for avoidance and capture of cigarette beetles and vinegar flies. Crop Prot. 2011, 30, 155-162. [CrossRef]

7. Matsuda, Y.; Kakutani, K.; Nonomura, T.; Kimbara, J.; Kusakari, S.; Osamura, K.; Toyoda, H. An oppositely charged insect exclusion screen with gap-free multiple electric fields. J. Appl. Phys. 2012, 112. [CrossRef]

8. Kakutani, K.; Matsuda, Y.; Haneda, K.; Nonomura, T.; Kimbara, J.; Kusakari, S.; Osamura, K.; Toyoda, H. Insects are electrified in an electric field by deprivation of their negative charge. Ann. Appl. Biol. 2012, 160, 250-259. [CrossRef]

9. Nonomura, T.; Matsuda, Y.; Kakutani, K.; Kimbara, J.; Osamura, K.; Kusakari, S.; Toyoda, H. Electrostatic measurement of dischargeable electricity and bioelectric potentials produced by muscular movements in flies. J. Electrostat. 2014, 72, 1-5. [CrossRef]

10. Takikawa, Y.; Matsuda, Y.; Kakutani, K.; Nonomura, T.; Kusakari, S.; Ito, H.; Osamura, K.; Toyoda, H. Electrostatic insect sweeper for eliminating whiteflies colonizing host plants; a complementary pest control device in an electric field screen-guarded greenhouse. Insects 2015, 6, 442-454. [CrossRef] [PubMed]

11. Matsuda, Y.; Nonomura, T.; Kakutani, K.; Kimbara, J.; Osamura, K.; Kusakari, S.; Toyoda, H. Avoidance of an electric field by insects: Fundamental biological phenomenon for an electrostatic pest-exclusion strategy. J. Phys. 2015, 646, 0120031-0120034. [CrossRef] 
12. Nonomura, T.; Matsuda, Y.; Kakutani, K.; Kimbara, J.; Osamura, K.; Kusakari, S.; Toyoda, H. An electric field strongly deters whiteflies from entering window-open greenhouses in an electrostatic insect exclusion strategy. Eur. J. Plant Pathol. 2012, 134, 661-670. [CrossRef]

13. Shimizu, K.; Matsuda, Y.; Nonomura, T.; Ikeda, H.; Tamura, N.; Kusakari, S.; Kimbara, J.; Toyoda, H. Dual protection of hydroponic tomatoes from rhizosphere pathogens Ralstonia solanacearum and Fusarium oxysporum f. sp. radicis-lycopersici and airborne conidia of Oidium neolycopersici with an ozone-generative electrostatic spore precipitator. Plant Pathol. 2007, 56, 987-997. [CrossRef]

14. Nonomura, T.; Matsuda, Y.; Kakutani, K.; Takikawa, Y.; Toyoda, H. Physical control of powdery mildew (Oidium neolycopersici) on tomato leaves by exposure to corona discharge. Can. J. Plant Pathol. 2008, 30, 517-524. [CrossRef]

15. Jonassen, N. Electrostatic effects and Abatement of static electricity. In Electrostatics; Kluwer Academic Publishers: Boston, MA, USA, 2002; pp. 75-120, ISBN 1-4020-7161-2.

16. Aplin, K.L.; Harrison, R.G. A computer-controlled Gerdien atmospheric ion counter. Rev. Sci. Instrum. 2000, 71, 3037-3041. [CrossRef]

17. Kakutani, K.; Matsuda, Y.; Haneda, K.; Sekoguchi, D.; Nonomura, T.; Kimbara, J.; Osamura, K.; Kusakari, S.; Toyoda, H. An electric field screen prevents captured insects from escaping by depriving bioelectricity generated through insect movements. J. Electrostat. 2012, 70, 207-211. [CrossRef]

18. Takikawa, Y.; Matsuda, Y.; Nonomura, T.; Kakutani, K.; Kimbara, J.; Osamura, K.; Kusakari, S.; Toyoda, H. Electrostatic guarding of bookshelves from mould-free preservation of valuable library books. Aerobiologia 2014, 30, 435-444. [CrossRef]

19. Cross, J.; Dielectrophoresis, A. Electrostatics: Principles, Problems and Applications; De Barr, A.E., Ed.; Adam Hilger: Bristol, PA, USA, 1987; pp. 269-276, ISBN-13: 978-0852745892.

20. Wegner, H.E. Electrical charging generators. In McGraw-Hill Encyclopedia of Science and Technology, 9th ed.; The Lakeside Press: New York, NY, USA, 2002; pp. 42-43, ISBN-13: 978-0079136657.

21. Katatani, A.; Mizuno, A. Generation of ionic wind by using parallel located flat plates. J. Inst. Electrostat. Jpn. 2010, 34, 187-192, ISSN 0386-2550. (In Japanese with English Abstract).

22. Halliday, D.; Resnick, R.; Walker, J. Electric fields. In Fundamentals of Physics; Johnson, S., Ford, E., Eds.; John Wiley \& Sons: New York, NY, USA, 2005; pp. 580-604, ISBN 0-471-23231-9. 